\title{
Psychosocial interventions for self-harm, suicidal ideation and suicide attempt in children and young people: What? How? Who? and Where?
}

\author{
Georgina Cox, Sarah Hetrick
}

Orygen, The National Centre of Excellence in Youth Mental Health, The University of Melbourne, Melbourne, Victoria, Australia

Correspondence to Dr Georgina Cox; georgina.cox@orygen.org.au

\begin{abstract}
We reviewed the evidence for the effectiveness of indicated individual psychosocial interventions for the treatment of self-harm, suicidal ideation and suicide attempts in children and young people, with a particular emphasis on the emerging use of electronic methods to deliver psychological interventions. In total, 16 randomised controlled trials (RCTs) were identified, none of which included children under the age of 12 years.

Cognitive-behavioural therapy is the most commonly implemented approach in RCTs until now, although problem-solving therapy, interpersonal psychotherapy, social support and distal support methods by provision of a green card and regular receipt of postcards have also been investigated. Young people have been recruited into RCTs within schools, outpatient clinics, emergency departments and inpatient facilities. Face-to-face delivery of therapy has dominated the intervention trials thus far; however, the use of the internet, social media and mobile devices to deliver interventions to young people and other family members allows for a more novel approach to suicide prevention in youth going forward.
\end{abstract}

\section{INTRODUCTION}

Suicidal ideation (SI), self-harm (SH), suicide attempt (SA) and suicide are significant public health concerns in young people. ${ }^{1}$ Suicide rates in this population have been rising, ${ }^{2}$ and suicide is now one of the leading causes of death in 15-29-year olds globally. ${ }^{3}$ In the age group 15-19 years, the suicide rate is estimated to be 7.4 per 100000 people, with rates in men being higher than in women (10.4 vs 4.1 per 100000 , respectively) ${ }^{4}$. There are limited data on the incidence of suicide in those under the age of 15 years; ${ }^{5}$ however, one study has estimated the mean rate of suicide in those up to 14 years to be $\sim 0.6$ per 100000 people worldwide. $^{7}$

SI, SH and SA are more common in young people than suicide. ${ }^{8} \mathrm{SI}$ can comprise thoughts of death or wishing to die and/or engaging in an $\mathrm{SA}$, and is a known risk factor for $\mathrm{SA}$ and death by suicide. ${ }^{9} 10$ Epidemiological data from community samples in the USA indicate that, in the past 12 months, $24 \%$ of young people between the ages of 12 and 17 years have experienced $\mathrm{SI}$, and between 10 and $17 \%$ have engaged in an SA. ${ }^{8}$ However, this prevalence is higher in clinical samples, with up to $85 \%$ of young people experiencing depressive symptoms also reporting SI. ${ }^{11}$ Furthermore, $32 \%$ of clinically referred children and young people will make an SA and by adulthood, between 2.5 and $7 \%$ will die as a result of suicide. ${ }^{11} 12$

There are a variety of different terms used when referring to $\mathrm{SH}$, with the terms 'deliberate or intentional self-harm' used widely in Europe and Australia. ${ }^{13} 14$ However, the prefix deliberate is being used less and less, as it can be seen as judgemental, and implies that a person is aware and in control of their actions, which may not always be the case. ${ }^{15}$ These terms are often used when determining if the presence or absence of suicidal intent is problematic. The term SA can also sometimes be used interchangeably when describing acts of SH. ${ }^{16}$ Generally, such behaviour includes cutting, scratching, hitting, burning the tissue on one's own body and selfpoisoning. For the purpose of this article, SH refers to behaviour where there is known SI, mixed motivations or SI cannot be determined. Community prevalence rates of $\mathrm{SH}$ in adolescents are estimated to be between 5 and $10 \%$ over a 12-month period, and rise to 16 to $22.8 \%$ over a lifetime..$^{131718} \mathrm{SH}$ is a known risk factor for SI and SA and death by suicide ${ }^{19}$ as well as being associated with a number of other negative outcomes such as mental health issues, poor educational and vocational outcomes and premature mortality due to other causes. ${ }^{20}{ }^{21}$ For the purpose of this review, SI, SH and SA will collectively be referred to as suicide-related behaviours (SRBs).
Interventions to prevent suicide can be classified using the Mrazek and Haggerty categories of universal, selective and indicated interventions. ${ }^{22}$ Universal interventions are delivered to the general public or to whole populations, not selected on the basis of increased risk (in this case, of suicide). Selective interventions are delivered to individuals or subgroups of the population whose risk of suicide might be higher than in the general population, but who do not yet show signs or symptoms (eg, young people with depressive symptoms or substance abuse problems). An indicated intervention is one that is targeted at people who have signs and symptoms, in this case, a history of SRBs.

\section{When might individual therapy be the best option?}

Individual, group-based and family oriented interventions are all treatment options for SRBs in young people. ${ }^{23} 24$ The family can be involved as a target of intervention by educating them about the signs of suicide in young people, involving them in planning for crises (eg, by reducing access to means) and assisting them to access services and attend appointments with their child. ${ }^{25}$ However, there are times when it is not possible to engage the family in therapy. Factors such as finding a time for therapy around work and school commitments, parents feeling overwhelmed by their child's symptoms, parents feeling blamed, judged or not listened to by therapists are some of the barriers encountered when engaging family in the therapeutic process. ${ }^{26}$

From a youth developmental perspective, young people often voice that they want privacy and confidentiality in therapy at a time when they are developing their independence. ${ }^{27-32}$ Often, individual therapy is what is sought by a young person and is what is appropriate given their age and stage. Family involvement can still be part of what clinicians aim to do in collaboration with the young person leg, at the very least letting the family know what is going on and ensuring that they are engaged as a support). Nevertheless, individual therapy may be the preferred option for young people and it is important to know which therapeutic approaches are effective for addressing SRBs as well as considering how and where such services are delivered.

Our aim in this review is to identify evidence for the effectiveness of individual psychosocial interventions on SRBs in children and young people up to the age of 25 years, with a particular focus on the type and setting of the intervention, as well as the method of delivery.

\section{Method}

PubMed, PsycINFO, Google Scholar and the Cochrane Library were searched from inception to January 2017 to locate English language systematic reviews (SRs) or meta-analyses (MAs) conducted within the 
context of an SR. SRs containing published or ongoing randomised controlled trials (RCTs) of individual psychosocial interventions delivered to children and/or young people up to the age of 25 years who had experienced SH, SI or an SA were included. SRs had to include trials where the primary or secondary outcome was an SRB or incidence of suicide. For the purpose of this review, trials could be included regardless of whether data were reported.

SRs that focused on the prevention of suicide in groups that had not reported any episodes of SRBs leg, universal and indicated interventions) or focused exclusively on non-suicidal self-injury were not included. Similarly, SRs that included trials where participants were recruited on the basis of a mental health diagnosis (eg, depression, borderline personality disorder) where an SRB was not a criterion for inclusion even if they measured an SRB were not included.

Trials where the primary therapeutic approach was family based, focused specifically on targeting family dynamics, but contained some individual sessions (eg, attachment-based family therapy) were not included. Trials where the therapeutic approach contained more than two family or group sessions were also excluded, as were trials where the intervention was a single therapeutic session which focused on the family. In the case of multiple publications on the same topic, the most recent or comprehensive publications were included.

The search terms applied were: suicid* AND self-harm* AND (child* OR young* OR adolescen* OR teen), along with meta* or review* in trial type in applicable search engines.

The following characteristics of trials were extracted: location of recruitment and location of intervention delivery, method of intervention delivery (eg, face-to-face, online), who delivered the intervention, type of therapeutic approach and comparison condition leg, treatment as usual (TAU)). Where possible, this information was extracted from the included SRs; however, in cases where this information was not available, we extracted it directly from the original trial publication.

\section{RESULTS}

Four SRs met the criteria for this review. ${ }^{23} 2433 \quad 34$ In many cases, these SRs included the same trials, however, owing to differences in the inclusion criteria, methods of analysis and the databases searched, it was decided that all four SRs were worthy of inclusion and exploration within this review. One of these SRs included psychosocial interventions for youth suicide across a number of settings and included RCTs targeting universal, selective and indicated populations; ${ }^{.33}$ only those delivered in indicated populations are discussed in this review. Two SRs focused on young people who had presented to clinical services as a result of $\mathrm{SH}^{17}$ or SRBs including passive $\mathrm{SI}^{23}$ and another SR focused on web-based and mobile psychosocial suicide interventions for young people. Three of the reviews included trials with young people aged between 12 and 25 years $^{23} 33{ }^{34}$ and one included trials with participants up to the age of 18 years. ${ }^{24}$ See table 1 for the characteristics of trials included in this review.

Sixteen individual trials from these four SRs are described within this review. One trial by Shand et al (2013), which was included as an unpublished trial in the SR by Perry et al (2016), appeared relevant during preparation of this review. However, this has now been published ${ }^{35}$ and the mean age of participants is 26.25 with a range of ages that extended up to 61, and is therefore excluded.

\section{What?}

The most common intervention approach tested in trials until now is cognitive-behavioural therapy (CBT), which has been tested in four trials $^{36-39}$ and is currently being tested in a further trial. ${ }^{40}$ Various protocols of CBT have been tested across these trials. Alavi et al (2013) used a waitlist comparison and tested a protocol of CBT that was designed to specifically address SRBs, based on the Stanley model. ${ }^{41}$
The main components of this include chain analysis (examines the circumstances, cognitions, emotions and behaviour leading up to and at the time of the SA), safety planning, psychoeducation, developing reasons for living and hope, as well as the more commonly used approaches of behavioural activation and cognitive restructuring, mood monitoring, emotion regulation and distress tolerance techniques, problem solving, goal setting, mobilising social support and assertiveness skills. Parents were allowed to participate in the first session and 'standard psychiatric care' with medication was used if needed, which was provided to both groups. Slee et al 2008 also implemented a protocol of CBT that specifically addressed $\mathrm{SH}$, but they developed the protocol themselves. It included chain analysis, cognitive restructuring, emotion regulation and distress tolerance techniques and problem solving in order to address the mechanisms that maintained $\mathrm{SH}$. Parents were encouraged to be involved as supports. CBT was delivered in the context of treatment as usual that included any of: psychotherapy, pharmacotherapy, alcohol/drug treatment, partial hospitalisation and community service and the comparison group received TAU alone. The ongoing trial by Robinson et al (2014) implements CBT which contains components specifically focused on SI.

While the explicit focus on SH was less clearly articulated in the publication of the LifeSPAN therapy conducted by Power et al (2003), it is described as including a collaborative risk assessment and formulation with a focus on a functional analysis of the suicidality. It included problem-solving training, psychoeducation for psychosis, emotional pain tolerance, stress management, self-esteem, help seeking and social skills training. Parents were not explicitly mentioned in the description of the intervention. Young people were also receiving TAU at a specialist early intervention clinic for first episode psychosis; and the comparison group in this trial received TAU alone.

The CBT protocol tested in the trial by Donaldson was called 'skillsbased treatment', which they describe as cognitive restructuring and behavioural strategies such as relaxation for affect management. It also included elements of problem-solving therapy (PST). Parents had brief contact with therapists to provide collateral information and there was one adjunct family session. This CBT protocol was compared with a supportive therapy.

Two trials have investigated a PST approach. ${ }^{42} 43$ Both trials followed the standard approach of D'Zurilla and Goldfried (1971) and D'Zurilla and Nezu (1999) which includes highly structured stages of problem solving. ${ }^{44}$ Specific skills are taught including: problem identification, goal setting, generating alternative solutions, decision-making (choosing a solution), solution implementation, assessment and verification (did the solution achieve goals?). However, it is worth noting that Fitzpatrick et al (2005) delivered the PST in one session via a 35-min video and the comparison group received a video about healthy lifestyles. In the trial by Eskin et al 2008, the PST was compared with a waitlist control condition. One further trial described their intervention as interpersonal PST (IPST). ${ }^{46}$ The IPST incorporated the stages of problem solving as described by D'Zurilla and Goldfried (1971) and it was compared with a brief problem-oriented approach. There was no family involvement in any of the PST approaches tested in these three trials.

Two trials have assessed the effectiveness of social support with TAU (which could include any of: psychotherapy, pharmacotherapy, alcohol/ drug treatment and partial hospitalisation) compared with TAU alone. ${ }^{47}{ }^{48}$ As well as receiving TAU, the intervention group had weekly supportive contact (discussing daily activities and concerns, encouraging activities in support of treatment goals) with someone they nominated as a support person. This nominated support person received 1.5 hours of psychoeducation about the young person's psychiatric disorders and treatment plan, risk factors for suicidal behaviour and warning signs for imminent risk, as well as strategies for communicating with young people. In the later trial in 2009, there were slight 
Table 1 Randomised controlled trials (RCTs) of indicated psychosocial interventions implemented with young people up to the age of 25 years

\begin{tabular}{|c|c|c|c|c|c|c|}
\hline Trial & Approach & Comparison condition & $\begin{array}{l}\text { Delivery } \\
\text { method }\end{array}$ & Delivered by & $\begin{array}{l}\text { Recruitment } \\
\text { setting }\end{array}$ & $\begin{array}{l}\text { Setting of } \\
\text { intervention }\end{array}$ \\
\hline Alavi et $a l^{36}$ & CBT & Waitlist & Face-to-face & $\begin{array}{l}\text { Clinician (no info in paper; } \\
\text { programme leader) }\end{array}$ & Inpatient & Unclear \\
\hline $\begin{array}{l}\text { Donaldson } \\
\text { et } a l^{37}\end{array}$ & CBT/PST & Supportive therapy & Face-to-face & $\begin{array}{l}\text { Five clinicians and two } \\
\text { individuals with master's } \\
\text { degrees }\end{array}$ & ED and inpatient & Outpatient \\
\hline $\begin{array}{l}\text { Eggert } \\
\text { et a }\left.\right|^{50}\end{array}$ & $\begin{array}{l}\text { MI(C-Care)+ } \\
\text { support }\end{array}$ & Brief screening assessment & Face-to-face & $\begin{array}{l}\text { Nurse, counsellor, social } \\
\text { worker }\end{array}$ & School & School \\
\hline Eskin et $a l^{42}$ & Problem solving & Waitlist & Face-to-face & $\begin{array}{l}\text { Two graduate students in } \\
\text { clinical psychology }\end{array}$ & $\begin{array}{l}\text { School and } \\
\text { university }\end{array}$ & Community \\
\hline $\begin{array}{l}\text { Fitzpatrick } \\
\text { et } a l^{43}\end{array}$ & Problem solving & Video on healthy lifestyles & Video & Researcher & $\begin{array}{l}\text { University and } \\
\text { community }\end{array}$ & $\begin{array}{l}\text { University and } \\
\text { community }\end{array}$ \\
\hline King et $a l^{47}$ & Social support & $\begin{array}{l}\text { TAU (could include psychotherapy, } \\
\text { pharmacotherapy, alcohol/drug treatment, partial } \\
\text { hospitalisation) }\end{array}$ & Face-to-face & Laypeople & Inpatient & Outpatient clinic \\
\hline $\begin{array}{l}\text { McLeavey } \\
\text { et al }\end{array}$ & $\begin{array}{l}\text { Interpersonal } \\
\text { problem solving }\end{array}$ & Brief problem-oriented approach & Face-to-face & $\begin{array}{l}\text { Clinical psychologists } \\
\text { and registrars in psychiatry }\end{array}$ & ED & Unclear \\
\hline Power et al ${ }^{38}$ & CBT & $\begin{array}{l}\text { TAU (treatment at specialist early intervention } \\
\text { clinic for first-episode psychosis) }\end{array}$ & Face-to-face & Psychologists & Outpatient clinic & Outpatient clinic \\
\hline $\begin{array}{l}\text { Robinson } \\
\text { et al }\end{array}$ & $\begin{array}{l}\text { Support and } \\
\text { self-help }\end{array}$ & $\begin{array}{l}\text { TAU (sources of help interview, and both groups } \\
\text { received whatever support they were receiving in } \\
\text { the community) }\end{array}$ & Postcard & Postcard & Outpatient clinic & Outpatient clinic \\
\hline $\begin{array}{l}\text { Robinson } \\
\text { et } a^{66}\end{array}$ & CBT & TAU & $\begin{array}{l}\text { Online (internet } \\
\text { based) }\end{array}$ & Online & School & School \\
\hline
\end{tabular}

CBT, cognitive-behavioural therapy; C-Care, counsellors care, assess, respond, empower; ED, emergency department; IPT, interpersonal psychotherapy; MI, motivational interview; PST, problem-solving therapy; TAU, treatment as usual.

adaptations to this intervention whereby only adults could be nominated as support people, and the interventions were for 6 months rather than 3 months. It should be noted that in the 2006 trial, in $61.5 \%$ of cases, the nominated support person was a parent. In the remainder of cases, the support person was a non-parent adult relative, a non-adult relative, unrelated peer, family friend or neighbour, teacher, school counsellor or administrator.

Another three trials also implemented a social support intervention (called C-Care: Counsellors Care, Assess, Respond, Empower) that also included a motivational interviewing approach. ${ }^{49-51}$ The intervention included a 2-hour computer-assisted assessment, a brief motivational counselling session with a focus on ensuring empathy towards the young person and to encourage help seeking; and an intervention to facilitate social network connections whereby young people were linked to a school-based case worker or their favourite teacher. There was some contact with parents to enhance support. The comparison group consisted of a brief screening assessment alone.

Interpersonal psychotherapy (IPT) was tested in one trial ${ }^{52}$ in which interpersonal problem domains of interpersonal conflict, interpersonal sensitivity and role transition and grief were targeted in relation to SI.
Some minimal family psychoeducation was included. The comparison group received usual school counselling.

One trial ${ }^{53}$ assessed the effectiveness of a distal support method, whereby adolescents who had been admitted to hospital for an episode of $\mathrm{SH}$ were given a token (hereafter called a green card), which they could use to be readmitted into hospital. The aim of the green card was to provide young people with 'an alternative way to escape from their environment and get help' (pg. 570). There was no family involvement. The intervention and the comparison group received TAU that consisted of the standard follow-up after discharge from hospital and treatment from their clinic or child psychiatry department.

Another trial ${ }^{54}$ used postcards, which were sent to participants on a monthly basis, and contained individual sources of help (identified in an initial 'sources of help interview' where young people were asked what they found useful in times of crisis, eg, listening to music, talking to a friend, etc), as well as evidence-based self-help strategies. The postcards also asked about the young person's well-being. There was no family involvement. The comparison group received the sources of help interview, and both groups received whatever support they were receiving in the community. 


\section{How?}

\section{CBT trials}

The majority of the trials that implemented CBT did so using a face-to-face method; ${ }^{36-39}$ however, one ongoing trial is delivering CBT through the internet. ${ }^{40}$

In the two trials delivering PST, one delivered the intervention face-to-face ${ }^{42}$ while the other used a 35 -minute video. ${ }^{43}$ Trials implementing supportive therapy and IPT, all did so using the face-to-face method. 474852

Two trials have used green cards ${ }^{53}$ or postcards ${ }^{54}$ to deliver their interventions, which did not involve any ongoing therapist contact.

\section{Where?}

In terms of the recruitment for trials, and ongoing location of the intervention, this varied considerably. Some trials recruited young people who had been admitted to the emergency department (ED), ${ }^{46}$ an inpatient mental health facility, $364748{ }^{36}$ both, $^{37}$ and outpatient mental health clinics. ${ }^{38} 3954$ Universities that contain research-based outpatient clinics have also been the location of recruitment. ${ }^{42} 43$

Of those recruited within an inpatient or ED facility, two of the interventions were delivered in the community following discharge ${ }^{47} 48$ and another focused on improving access to services in the future. ${ }^{53}$ In the trials by Alavi and McLeavey, the treatment setting is not explicitly stated; however, they appear to be delivered in an outpatient setting. Trials that recruited within an outpatient setting (hospital based or university based) delivered treatment on an outpatient basis.

A limited number of trials have recruited within a school setting, and subsequently delivered interventions at school. ${ }^{40} 49-52$

\section{Who?}

Interventions varied in terms of whom they were delivered by, often as a function of the setting. In school settings, nurses, school counsellors and social workers were all cited as delivering interventions to young people, ${ }^{49-51}$ as well as lay support people. ${ }^{47} 48$ In the ongoing study by Robinson et al (2014), school counsellors assist in delivering the CBT-based internet intervention. In order to deliver these interventions, training was generally provided by the research team.

Within inpatient, outpatient and ED settings, clinicians with varying experience have generally implemented CBT, IPT and PST. Clinicians have included those currently in training as clinical psychologists or psychiatrists (at a master's or doctoral level equivalent), as well as clinicians experienced in delivering CBT with clients who engage in $\mathrm{SH}^{39}$ and licensed clinical psychologists.

\section{What has been effective?}

Trials that used a CBT that specifically targeted SH have shown promising results. In the trial conducted by Slee et al in 2008, there were reductions in the number of $\mathrm{SH}$ episodes at 9-months follow-up and a significant reduction in the severity of $\mathrm{SI}$ at all time points compared with the TAU group. In the trial conducted by Alavi et al in 2013, there were significant differences in SI between participants receiving CBT focused on suicidality and those in the waitlist group. In the trials conducted by Power et al (2003) and Donaldson et al (2005), there were no differences in outcomes.

There were three trials of PST. In two of these, ${ }^{42}{ }^{43}$ there were significant impacts of the intervention in the non-clinical samples experiencing SI, though not across all outcomes. In the trial conducted by Eskin et al (2008), there were differences in SI post intervention compared with a waitlist control group. Likewise, in the trial conducted by Fitzpatrick, there were significant differences in SI at on1-month follow-up compared with those who received a video about health lifestyles. In the trial conducted by McLeavey, there were no differences in $\mathrm{SA}$ at 12 months (and no other suicide-related outcomes).
In the trials conducted by King et al (2006, 2009), there were no significant differences in SI or in the percentage of participants who made an SA in the follow-up period between the group that received support and TAU and the group that received TAU alone. In the earlier trial, the authors did note some positive effects in girls, and in the later trial they commented that there was a more rapid reduction in SI for those with a history of multiple SAs.

Of the three trials of school-based C-Care trials, there was only one significant finding with differences shown between groups in SI. ${ }^{51}$ There were no differences in $\mathrm{SI}$ in the other trials and no differences in SA across the three trials. In the IPT school-based trial, ${ }^{52}$ participants reported significant reductions in SI compared with those who received standard school counselling services.

The provision of an emergency green card ${ }^{53}$ did not result in significant differences in repetition of SA, and similarly, the receipt of postcards ${ }^{54}$ did not impact on SRBs.

\section{DISCUSSION}

This clinical review identified 16 RCTs from four SRs that have investigated the effectiveness of individual psychosocial interventions for SRBs. $^{23} 243334$ Generally, the sample sizes of the trials were small and thus lacked power. There were a variety of differences in where young people were recruited from, what intervention was delivered and by whom across the studies. The outcome measures that were used differed across the trials, as well as the follow-up assessment points, making it challenging to draw conclusions across trials. This echoes the conclusions of the SRs included in this review. Despite the incidence of suicide in children under the age of $12,{ }^{6}$ no trials located targeted children in the younger age range. As has been noted in the wider literature on suicide prevention, generating evidence in this area has been hampered by a number of ethical and practical concerns, including small sample sizes, underpowered trials due to the overall low rate of suicide, as well as the varying definitions of SRBs used in studies to select participants. ${ }^{55} 56$

\section{What?}

The 16 trials described within this review have implemented CBT, PST, IPT, social support and distal support methods by provision of a green card and regular receipt of postcards. At present, the most commonly investigated individual therapeutic approach identified in the RCTs was CBT. This mirrors other areas of mental health such as in depression. ${ }^{57} 58$

The SR by Robinson et al (2011) concluded that CBT appears to have promise in reducing SRBs in young people who present specifically to clinical services. However, the SR by Hawton et al (2015) found no evidence of effect of CBT on repetition of SH. The conclusions in both these SRs were based on single trials. A further trial adds some weight to the potential efficacy of CBT in clinical populations, particularly CBT that specifically targets SRBs. This is consistent with a recent review that showed that interventions that specifically target these behaviours, rather than the symptoms associated with this behaviour like depression, hopelessness and quality of life, were more effective at reducing these behaviours. ${ }^{59}$ Further, given the effectiveness of CBT in the treatment of disorders that are associated with SRBs, such as depression, ${ }^{6061}$ it may be prudent to continue to explore this avenue. Also of note is that Hawton et al suggested that dialectical behaviour therapy (DBT) and mentalisation-based treatment (MBT) were worthy of further investigation. Within this review, there were no trials that implemented these therapeutic approaches in a purely individual format (both have been trialled in a group format, or had a degree of family involvement). There is also evidence suggesting that DBT and MBT are effective in reducing $\mathrm{SI}$ in adults. ${ }^{62}$ As such, there may be scope to modify these interventions for individual delivery with suicidal youth. 
Although the SR conducted by Calear et al did not report on the type of psychotherapy that was most effective, they did analyse interventions delivered to individuals, versus those delivered to families and groups. They concluded that interventions delivered to individuals alone were effective in reducing $\mathrm{Sl}$, whereas group and family programmes had effects on SAs only. They also report that interventions that include an individual and family component appear to affect SI and SA. Our review sought to include trials delivered to individuals alone, without a family focus or more than two sessions involving a family member. Some CBT trials and the one IPT trial included minimal family involvement and, where this was the case, it tended to be a single session at the beginning of the intervention, in order to gain collateral information and/or to provide psychoeducation and engage parents as supports. ${ }^{47} 48$ Of note was the social support intervention where young people often chose their parent as the nominated support person, which, while not effective in terms of reductions in SRBs, highlights that including parents as a support may often be acceptable to young people. Clinicians need to balance the autonomy of the young person and their preference for family involvement, with the potential benefits and ethical issues inherent in managing suicide risk when delivering individual therapy.

The comparison conditions implemented in trials generally included TAU, or another active intervention, given the ethical concerns in withholding treatment from those at risk of suicide. ${ }^{56}$ TAU was often a high level of quality clinical care, for example, in the trial by Power et al, where the intervention was given in the context of high-quality standard clinical care of young people with psychosis by experienced clinicians. Such comparison groups, although ethically important, may limit the degree to which differences can be found between groups.

\section{How, who and where?}

Young people have been recruited into trials within schools, outpatient clinics, EDs and inpatient facilities. Generally, the intervention was delivered in the same place as where young people were recruited (with the exception of EDs). Further, the overwhelming majority of the trials used face-to-face methods to deliver interventions. These were delivered by a variety of professionals, from clinicians experienced in working with those engaging with $\mathrm{SH}$ in clinical settings, to school counsellors and laypeople in school and community settings. None of the SRs located within this review explored whether these aspects of where people are recruited from, how the intervention is delivered, and who by moderated treatment outcome, most likely due to the lack of RCTs in this area.

Of note are the relatively few examples of the use of technology to deliver interventions targeting suicidal young people, ${ }^{40}$ despite the sharp rise in the use of technology to deliver mental health services across the lifespan. ${ }^{63}$ As Perry et al (2016) noted in their SR, other areas of suicide prevention, such as screening young people for risk of suicide, are beginning to use web-based platforms to deliver services. ${ }^{64}$ The use of 'real time' monitoring and intervention provided via handheld devices to support young people 'in the moment' has been flagged as important for overcoming difficulties mental health services have with responding to fluctuating $\mathrm{SI}$ and episodes of $\mathrm{SH}$ and $\mathrm{SA} .{ }^{65}$ Despite the trial by Tighe and colleagues not being eligible for inclusion in this review, the lbobbly intervention is worthy of further exploration in an adolescent and youth population (under 25 years) given its accessibility. ${ }^{35}$

The use of social media has also been highlighted as having the potential to support young people with SRBs. ${ }^{66} \mathrm{~A}$ recent publication by Rice et al (2016) describes the implementation of a handful of online and social media interventions for young people at risk of suicide, as well as how such interventions may act to 'bolster social connectedness'. ${ }^{67}$ Accessing support, whether in the form of interventions, or contact with others, inbetween sessions may be beneficial for young people experiencing SRBs. It would be interesting to explore which of the most effective interventions or elements of interventions for reducing SI, SA and SH could be implemented as interventions delivered via handheld devices like smartphones. For example, DBT commonly involves teaching distress tolerance, an element of intervention that may be incorporated into a mobile app. A particular feature of the work done in this area is a focus on participatory design, including engagement of young people as codesigners, in order to ensure that the views and preferences of these end users are taken into account in the design. This is crucial to ensure the acceptability of the intervention, which in turn is likely to enhance uptake and engagement. ${ }^{68}$

Online platforms may also provide an opportunity for family intervention, without having to compromise a young person's preference for individual therapy. For example, a web-based support component for parents could be delivered alongside individual therapy for the young person, to provide them with basic psychoeducation around suicide in youth, including strategies to enable them to better support their child. In the area of psychosis, online family support interventions are beginning to be developed. ${ }^{69}$

There have been obvious concerns about use of technology-based interventions in terms of who and how to deal with risk. Any intervention delivered to young people experiencing SRBs in the online environment must be able to respond to risk. This can be done, provided there is appropriate moderation of the site, through thorough safety protocols. ${ }^{67}$

\section{CONCLUSIONS}

Many of the trials identified within this review failed to find significant differences in SI, SA and SH outcomes between groups, and as we have not sought to conduct an MA, it makes it challenging to recommend any specific therapeutic approach over another. Research is urgently needed in this area, and given the potential effectiveness of $\mathrm{CBT}$, DBT and MBT in treating SI and SH in other population groups, these are avenues worthy of further exploration. Although face-to-face delivery of therapy has dominated the intervention trials thus far, the use of the internet, social media and mobile devices in young people allows for innovative and novel approaches to suicide prevention in youth. Designing interventions with young people, which can be delivered in real time to young people, may be useful, as well as using electronic devices to support parents as an adjunct to individual therapy with young people.

Twitter Follow Georgina Cox@g_rose_cox

Competing interests None declared.

Provenance and peer review Not commissioned; externally peer reviewed.

doi:10.1136/eb-2017-102667

Received 10 March 2017; Accepted 13 March 2017

\section{REFERENCES}

1. Gore FM, Bloem PJ, Patton GC, et al. Global burden of disease in young people aged 10-24 years: a systematic analysis. Lancet 2011;377:2093-102.

2. Viner RM, Coffey $C$, Mathers $C$, et al. 50 -year mortality trends in children and young people: a study of 50 low-income, middle-income, and high-income countries. Lancet 2011;377:1162-74.

3. World Health Organization (WHO). Preventing Suicide: A global imperative. Geneva: WHO, 2014.

4. Wasserman D, Cheng 01 , Jiang GX. Global suicide rates among young people aged 15-19. World Psychiatry 2005;4:114-20.

5. Dilillo D, Mauri S, Mantegazza C, et al. Suicide in pediatrics: epidemiology, risk factors, warning signs and the role of the pediatrician in detecting them. Ital J Pediatr 2015;41:49.

6. Soole R, Kolves K, De Leo D. Suicide in children: a systematic review. Arch Suicide Res 2015;19:285-304.

7. Dervic K, Brent DA, Oquendo MA. Completed suicide in childhood. Psychiatr Clin North Am 2008;31:271-91. 
8. Nock MK, Borges G, Bromet EJ, et al. Cross-national prevalence and risk factors for suicidal ideation, plans, and attempts. Br J Psychiatry 2008;192:98-105

9. Cash SJ, Bridge JA. Epidemiology of youth suicide and suicidal behavior. Curr Opin Pediatr 2009;21:613-9.

10. Miranda R, Ortin A, Scott M, et al. Characteristics of suicidal ideation that predict the transition to future suicide attempts in adolescents. J Child Psychol Psychiatry 2014;55:1288-96.

11. Kovacs M, Goldston D, Gatsonis C. Suicidal behaviors and childhood-onset depressive disorders: a longitudinal investigation. J Am Acad Child Adolesc Psychiatry 1993;32:8-20.

12. Harrington R, Bredenkamp D, Groothues C, et al. Adult outcomes of childhood and adolescent depression. III. Links with suicidal behaviours. J Child Psychol Psychiatry 1994;35:1309-19.

13. Madge N, Hewitt A, Hawton $\mathrm{K}$, et al. Deliberate self-harm within an international community sample of young people: comparative findings from the Child $\varepsilon$ Adolescent Self-harm in Europe (CASE) Study. J Child Psychol Psychiatry 2008;49:667-77

14. Muehlenkamp JJ, Claes L, Havertape L, et al. International prevalence of adolescent non-suicidal self-injury and deliberate self-harm. Child Adolesc Psychiatry Ment Health 2012;6:10

15. Tolmunen T, Rissanen ML, Hintikka J, et al. Dissociation, self-cutting, and other self-harm behavior in a general population of Finnish adolescents. J Nerv Ment Dis 2008;196:768-71.

16. Skegg K. Self-harm. Lancet 2005;366:1471-83.

17. Hawton K, Rodham $K$, Evans $E$, et al. Deliberate self harm in adolescents: self report survey in schools in England. BMJ 2002;325:1207-11.

18. Patton GC, Hemphill SA, Beyers JM, et al. Pubertal stage and deliberate self-harm in adolescents. J Am Acad Child Adolesc Psychiatry 2007;46:508-14.

19. Suominen K, Isometsä E, Suokas J, et al. Completed suicide after a suicide attempt: a 37-year follow-up study. Am J Psychiatry 2004;161:562-3.

20. Mars B, Heron J, Crane C, et al. Clinical and social outcomes of adolescent self harm: population based birth cohort study. BMJ 2014;349:g5954.

21. Bergen H, Hawton K, Waters K, et al. Premature death after self-harm: a multicentre cohort study. Lancet 2012;380:1568-74.

22. Mrazek PB, Haggerty RJ. Reducing risks for mental disorders : frontiers for preventive intervention research. Washington DC: National Academy Press, 1994:605, xxvii.

23. Robinson J, Hetrick SE, Martin C. Preventing suicide in young people: systematic review. Aust N Z J Psychiatry 2011:45:3-26.

24. Hawton K, Witt KG, Taylor Salisbury TL, et al. Interventions for self-harm in children and adolescents. Cochrane Database Syst Rev 2015;(12):CD012013.

25. Daniel SS, Goldston DB. Interventions for suicidal youth: a review of the literature and developmental considerations. Suicide Life Threat Behav 2009;39:252

26. Baker-Ericzén MJ, Jenkins MM, Haine-Schlagel R. Therapist, parent, and youth perspectives of treatment barriers to family-focused community outpatient mental health services. J Child Fam Stud 2013;22:854-68.

27. James AM. Principles of youth participation in mental health services. Med J Aust 2007;187(7 Suppl):S57-60.

28. Tylee A, Haller D, Graham T, et al. Youth-friendly primary-care services: how are we doing and what more needs to be done? Lancet 2007;369:1565-73.

29. Booth ML, Bernard D, Quine S, et al. Access to health care among Australian adolescents young people's perspectives and their sociodemographic distribution. $J$ Adolesc Health 2004:34:97-103.

30. Klein JD, Mcnulty M, Flatau CN. Adolescents' access to care: teenagers' self-reported use of services and perceived access to confidential care. Arch Pediat Adolesc Med 1998;152:676-82.

31. Anderson JE, Lowen CA. Connecting youth with health services: systematic review. Can Fam Physician 2010;56:778-84

32. Jacobson L, Richardson G, Parry-Langdon N, et al. How do teenagers and primary healthcare providers view each other? An overview of key themes. $\mathrm{Br} J$ Gen Pract 2001;51:811-16.

33. Calear AL, Christensen $\mathrm{H}$, Freeman A, et al. A systematic review of psychosocial suicide prevention interventions for youth. Eur Child Adolesc Psychiatry 2016;25:467-82.

34. Perry $\mathbf{Y}$, Werner-Seidler A, Calear AL, et al. Web-based and mobile suicide prevention interventions for young oeople: a systematic review. J Can Acad Child Adolesc Psychiatry 2016;25:73-9.

35. Tighe J, Shand F, Ridani R, et al. Ibobbly mobile health intervention for suicide prevention in Australian indigenous youth: a pilot randomised controlled trial. BMJ Open 2017; 7:e013518.

36. Alavi A, Sharifi B, Ghanizadeh A, et al. Effectiveness of cognitive-behavioral therapy in decreasing suicidal ideation and hopelessness of the adolescents with previous suicidal attempts. Iran J Pediatr 2013;23:467-72.

37. Donaldson D, Spirito A, Esposito-Smythers C. Treatment for adolescents following a suicide attempt: results of a pilot trial. J Am Acad Child Adolesc Psychiatry 2005; $44: 113-20$

38. Power PJR, Bell RJ, Mills R, et al. Suicide prevention in first episode psychosis: the development of a randomised controlled trial of cognitive therapy for acutely suicidal patients with early psychosis. Aust N Z J Psychiatry 2003:37:414-20.
39. Slee N, Garnefski N, Van Der Leeden R, et al. Cognitive-behavioural intervention for self-harm: randomised controlled trial. Br J Psychiatry 2008;192:202-11.

40. Robinson J, Hetrick S, Cox G, et al. The development of a randomised controlled trial testing the effects of an online intervention among school students at risk of suicide. BMC Psychiatry 2014:14:155

41. Stanley B, Brown G, Brent DA, et al. Cognitive-behavioral therapy for suicide prevention (CBT-SP): treatment model, feasibility, and acceptability. J Am Acad Child Adolesc Psychiatry 2009;48:1005-13.

42. Eskin M, Ertekin K, Demir H. Efficacy of a problem-solving therapy for depression and suicide potential in adolescents and young adults. Cogn Ther Res 2008:32:227-45.

43. Fitzpatrick KK, Witte TK, Schmidt NB. Randomized controlled trial of a brief problem-orientation intervention for suicidal ideation. Behav Ther 2005:36:323-33.

44. D'Zurilla TJ, Goldfried MR. Problem solving and behavior modification. J Abnorm Psychol 1971:78:107-26.

45. D'Zurilla TJ, Nezu AM. Problem-solving therapy: a social competence approach to clinical intervention. 2nd edn. New York: Springer, 1999.

46. Mcleavey BC, Daly RJ, Ludgate JW, et al. Interpersonal problem-solving skills training in the treatment of self-poisoning patients. Suicide Life Threat Behav 1994;24:382-94.

47. King CA, Klaus N, Kramer A, et al. The youth-nominated support team-version II for suicidal adolescents: a randomized controlled intervention trial. J Consult Clin Psychol 2009;77:880-93.

48. King CA, Kramer A, Preuss $L$, et al. Youth-nominated support team for suicidal adolescents (Version 1): a randomized controlled trial. J Consult Clin Psychol 2006:74:199-206.

49. Hooven C, Walsh E, Pike KC, et al. Promoting CARE: including parents in youth suicide prevention. Fam Community Health 2012;35:225-35.

50. Eggert LL, Thompson EA, Randell BP, et al. Preliminary effects of brief school-based prevention approaches for reducing youth suicide--risk behaviors, depression, and drug involvement. J Child Adolesc Psychiatr Nurs 2002;15:48-64.

51. Thompson EA, Eggert LL, Randell BP, et al. Evaluation of indicated suicide risk prevention approaches for potential high school dropouts. Am J Public Health 2001;91:742-52

52. Tang TC, Jou SH, Ko CH, et al. Randomized study of school-based intensive interpersonal psychotherapy for depressed adolescents with suicidal risk and parasuicide behaviors. Psychiatry Clin Neurosci 2009:63:463-70.

53. Cotgrove AJ, Zirinsky L, Black D, et al. Secondary prevention of attempted suicide in adolescence. Journal of Adolescence 1995;18:569-77.

54. Robinson J, Yuen HP, Gook S, et al. Can receipt of a regular postcard reduce suicide-related behaviour in young help seekers? A randomized controlled trial. Early Interv Psychiatry 2012;6:145-52.

55. Saunders KE, Smith KA. Interventions to prevent self-harm: what does the evidence say? Evid Based Ment Health 2016

56. Lakeman R, Fitzgerald M. The ethics of suicide research. Crisis 2009;30:13-9.

57. Hofmann SG, Asnaani A, Vonk IJJ, et al. The efficacy of cognitive behavioral therapy: a review of meta-analyses. Cognit Ther Res 2012;36:427-40.

58. Callahan P, Liu P, Purcell $R$, et al. Evidence map of prevention and treatment interventions for depression in young people. Depress Res Treat 2012;2012:820735.

59. Meerwijk EL, Parekh A, Oquendo MA, et al. Direct versus indirect psychosocial and behavioural interventions to prevent suicide and suicide attempts: a systematic review and meta-analysis. Lancet Psychiatry 2016;3:544-54.

60. Weisz JR, Gray JS. Evidence-based psychotherapy for children and adolescents: data from the present and a model for the future. Child and Adolescent Mental Health 2008:13:54-65.

61. Watanabe N, Hunot V, Omori IM, et al. Psychotherapy for depression among children and adolescents: a systematic review. Acta Psychiatr Scand 2007;116:84-95.

62. Panos PT, Jackson JW, Hasan 0, et al. Meta-analysis and systematic review assessing the efficacy of dialectical behavior therapy (DBT). Res Soc Work Pract 2014;24:213-23.

63. Proudfoot $\mathbf{J}$. The future is in our hands: the role of mobile phones in the prevention and management of mental disorders. Aust N Z J Psychiatry 2013:47:111-3.

64. King CA, Eisenberg D, Zheng $K$, et al. Online suicide risk screening and intervention with college students: a pilot randomized controlled trial. J Consult Clini Psychol 2015;83:630-6

65. Hetrick SE, Goodall J, Yuen HP et al. Comprehensive online self-monitoring to support clinicians manage risk of suicide in youth depression. Crisis 2016:1-11.

66. Robinson J, Cox G, Bailey E, et al. Social media and suicide prevention: a systematic review. Early Interv Psychiatry 2016;10:103-21.

67. Rice S, Robinson J, Bendall S, et al. Online and social media suicide prevention interventions for young people: a focus on implementation and moderation. J Can Acad Child Adolesc Psychiatry 2016;25:80-6.

68. Wadley G, Lederman R, Gleeson J, et al. Participatory design of an online therapy for youth mental health, in proceedings of the 25th Australian Computer-Human Interaction Conference. Augmentation, Application, Innovation, Collaboration. Adelaide, Australia: ACM, 2013:517-526.

69. Alvarez-Jimenez M, Alcazar-Corcoles MA, GonzãiLez-Blanch C, et al. Online, social media and mobile technologies for psychosis treatment: a systematic review on novel user-led interventions. Schizophr Res 2014:156:96-106. 